PROCEEDINGS OF THE

AMERICAN MATHEMATICAL SOCIETY

Volume 137, Number 8, August 2009, Pages 2567-2574

S 0002-9939(09)09806-2

Article electronically published on February 20, 2009

\title{
INEQUALITIES BETWEEN RANKS AND CRANKS
}

\author{
KATHRIN BRINGMANN AND KARL MAHLBURG
}

(Communicated by Ken Ono)

\begin{abstract}
Higher moments of the partition rank and crank statistics have been studied for their connections to combinatorial objects such as Durfee symbols, as well as for their connections to harmonic Maass forms. This paper proves the first several cases of (and strengthens) a conjecture due to Garvan, which states that the moments of the crank function are always larger than the moments of the rank function. Furthermore, asymptotic estimates for these differences are also proven.
\end{abstract}

\section{Introduction AND STATEMENT OF RESUlts}

The deceptively simple combinatorial generating functions found in the theory of partitions have seen a great deal of interest in recent years, as they lie directly in the intersection of basic hypergeometric series and automorphic forms. This has allowed the use of techniques taken from combinatorics, $q$-series, and complex analysis, and has led to a number of important results about harmonic Maass forms and mock theta functions [8, 10, 11, 12. Many of these modern results share a common ancestor in Ramanujan's original observations on the arithmetic of the partition function $p(n)$ [17, 18. Most famously, the three "Ramanujan congruences" state that if $\ell \in\{5,7,11\}$, then $p\left(\ell n+\delta_{\ell}\right) \equiv 0(\bmod \ell)$ for all $n \geq 0$, where $24 \delta_{\ell} \equiv 1$ $(\bmod \ell)$.

Hoping to understand the Ramanujan congruences from a combinatorial perspective, Dyson defined the partition rank function to be the largest part minus the number of parts [14. Atkin and Swinnerton-Dyer [7] later proved that Dyson's rank provides a combinatorial explanation of the congruences modulo 5 and 7 , but not the congruence modulo 11, leading Dyson to conjecture the existence of a more satisfactory statistic. Subsequently, Andrews and Garvan found the partition crank, which successfully dissects all three congruences simultaneously (see [4] for the precise combinatorial definition).

In order to better understand these two statistics, we will use analytic methods on the generating functions for the crank and rank. Let $\mathcal{M}(m, n)(\operatorname{resp} . \mathcal{N}(m, n))$ denote the number of partitions of $n$ with crank (resp. rank) $m$. Ignoring a minor error in the definition of $\mathcal{M}(m, n)$ when $n=1$, the two-parameter generating

Received by the editors October 9, 2008, and, in revised form, October 20, 2008.

2000 Mathematics Subject Classification. Primary 11P81; Secondary 05A17.

The first author was partially supported by NSF grant DMS-0757907.

The second author was partially supported by NSA Grant 6917958 .

(C)2009 American Mathematical Society Reverts to public domain 28 years from publication 
functions may be written as [4, 7]

$$
\begin{aligned}
C(x ; q):=\sum_{\substack{m \in \mathbb{Z} \\
n \geq 0}} \mathcal{M}(m, n) x^{m} q^{n} & =\prod_{n \geq 1} \frac{1-q^{n}}{\left(1-x q^{n}\right)\left(1-x^{-1} q^{n}\right)} \\
& =\frac{1-x}{(q)_{\infty}} \sum_{n \in \mathbb{Z}} \frac{(-1)^{n} q^{n(n+1) / 2}}{1-x q^{n}}, \\
R(x ; q):=\sum_{\substack{m \in \mathbb{Z} \\
n \geq 0}} \mathcal{N}(m, n) x^{m} q^{n} & =\sum_{n \geq 0} \frac{q^{n^{2}}}{(x q ; q)_{n}\left(x^{-1} q ; q\right)_{n}} \\
& =\frac{1-x}{(q)_{\infty}} \sum_{n \in \mathbb{Z}} \frac{(-1)^{n} q^{n(3 n+1) / 2}}{1-x q^{n}} .
\end{aligned}
$$

Remark. Although the final expressions for $C(x ; q)$ and $R(x ; q)$ appear quite similar, their analytic behavior is markedly different. For example, if $x \neq 1$ is a fixed root of unity, then $C(x ; q)$ is (essentially) a meromorphic modular form [16, whereas $R(x ; q)$ is (essentially) the holomorphic part of a harmonic Maass form [12. It is therefore somewhat surprising that we are able to compare the coefficients $\mathcal{M}(m, n)$ and $\mathcal{N}(m, n)$ with the precision described below.

In addition to the crank and rank statistics, we are also interested in their weighted moments. In particular, for even integers $k$, define the $k$-th rank (resp. crank) moments as

$$
\begin{aligned}
M_{k}(n) & :=\sum_{m \in \mathbb{Z}} m^{k} \mathcal{M}(m, n), \\
N_{k}(n) & :=\sum_{m \in \mathbb{Z}} m^{k} \mathcal{N}(m, n) .
\end{aligned}
$$

Both the crank and rank moments vanish when $k$ is odd due to the symmetries of the statistics $[6]$.

A number of recent works highlight the importance of these moments within the theory of partitions and congruences; for example, studies of the smallest parts partition function $\operatorname{spt}(n)$ [2] and Durfee symbols [3, 10] relied heavily on the intrinsic combinatorial connections to crank and rank moments. Our main focus in this note is unpublished observations of Garvan regarding the relative size of the crank and rank moments.

Conjecture (Garvan). Let $k \geq 2$ be an even integer.

(1) For all $n \geq 2$, we have $M_{k}(n)>N_{k}(n)$.

(2) As $n \rightarrow \infty$, we have $M_{k}(n) \sim N_{k}(n)$.

Remark. The case $k=2$ follows from work of Andrews [2], who showed directly from the generating functions that the smallest parts partition function satisfies

$$
\operatorname{spt}(n)=\frac{1}{2}\left(M_{2}(n)-N_{2}(n)\right)>0 .
$$

Remark. Garvan's conjecture can be interpreted as stating that the distribution of the crank statistic is slightly "wider" than that of the rank, but not significantly so. This is unexpected, as there is little about the combinatorial definitions of the crank and rank that suggest any close relations. 
We believe that a much stronger statement holds, and adopt the following notation for the difference between the crank and rank moments:

$$
D_{k}(n):=M_{k}(n)-N_{k}(n) .
$$

Conjecture 1.1. Suppose that $k \geq 2$ is even.

(1) As $n \rightarrow \infty$,

$$
M_{k}(n) \sim N_{k}(n) \sim \alpha_{k} \cdot n^{\frac{k}{2}} p(n),
$$

where $\alpha_{k} \in \mathbb{Q} \geq 0$.

(2) Garvan's inequality holds for all $n$, and as $n \rightarrow \infty$,

$$
D_{k}(n) \sim \beta_{k} n^{\frac{(k-1)}{2}} \cdot p(n),
$$

where $\beta_{k} \in \frac{\sqrt{6}}{\pi} \mathbb{Q}$ is positive.

In this paper, we prove asymptotic formulae that confirm the plausibility of the conjecture.

Theorem 1.2. For even $k \geq 2$, there exist rational constants $\alpha_{k}, \alpha_{k}^{\prime}$ such that

$$
\begin{aligned}
M_{k}(n) & \sim \alpha_{k} \cdot n^{\frac{k}{2}} p(n), \\
N_{k}(n) & \sim \alpha_{k}^{\prime} \cdot n^{\frac{k}{2}} p(n) .
\end{aligned}
$$

It is difficult to write down general formulas for $\alpha_{k}$ and $\alpha_{k}^{\prime}$, which is the chief obstacle in proving the conjecture for all $k$. However, we calculate these constants explicitly in order to (essentially) prove the first two cases of the conjecture.

Theorem 1.3. If $k=2,4$, then Conjecture 1.1 holds for sufficiently large $n$.

Remark. It should be possible to extend the inequalities from part (2) of Garvan's conjecture to all $n$ using known methods, although the estimates required are rather technical (see 8] for a related example and [11] for a more general setting).

The bulk of the paper is in Section 2, where we prove Theorem 1.3 (Theorem 1.2 will follow as a corollary of the arguments used in the proof). Section 3 follows with some concluding remarks about partition combinatorics.

\section{RANK-CRANK RELATIONS AND PROOFS}

We begin with the case $k=2$ and determine the asymptotic behavior of $D_{2}(n)$, providing the exact values for the constants from Conjecture 1.1.

Theorem 2.1. As $n \rightarrow \infty$,

$$
M_{2}(n) \sim N_{2}(n) \sim 2 n p(n) .
$$

Moreover, as $n \rightarrow \infty$,

$$
D_{2}(n) \sim \frac{2}{(24 n-1)^{\frac{1}{4}}} I_{\frac{1}{2}}(y) \sim \frac{2 \sqrt{6 n}}{\pi} p(n),
$$

where $I_{n}$ denotes the (modified) Bessel function of order $n$ and

$$
y_{n}:=\frac{\pi \sqrt{24 n-1}}{6} .
$$


Proof. Works of Dyson [15] and Andrews [2] show, respectively, that

$$
\begin{aligned}
M_{2}(n) & =2 n p(n), \\
N_{2}(n) & =2 \eta_{2}(n),
\end{aligned}
$$

where $\eta_{2}(n)$ is the number of 2-marked Durfee symbols of $n$. Moreover, as $n \rightarrow \infty$ (see [1] Chapter 5),

$$
p(n) \sim \frac{2 \pi}{(24 n-1)^{\frac{3}{4}}} I_{\frac{3}{2}}\left(y_{n}\right) .
$$

Furthermore, we obtain from 9]

$$
\eta_{2}(n) \sim-\frac{3}{2(24 n-1)^{\frac{1}{4}}} I_{\frac{1}{2}}\left(y_{n}\right)+\frac{\pi(24 n-1)^{\frac{1}{4}}}{12} I_{-\frac{1}{2}}\left(y_{n}\right)+\frac{\pi}{12(24 n-1)^{\frac{3}{4}}} I_{\frac{3}{2}}\left(y_{n}\right) .
$$

This gives

$D_{2}(n) \sim \frac{2 \pi}{(24 n-1)^{\frac{3}{4}}}\left(\frac{(24 n-1)}{12} I_{\frac{3}{2}}\left(y_{n}\right)-\frac{(24 n-1)}{12} I_{-\frac{1}{2}}\left(y_{n}\right)\right)+\frac{3}{(24 n-1)^{\frac{1}{4}}} I_{\frac{1}{2}}\left(y_{n}\right)$.

We now use the general Bessel function relation (see 5 for this and other basic facts)

$$
I_{a+1}(y)-I_{a-1}(y)=-\frac{2 a}{y} I_{a}(y),
$$

which specializes to

$$
I_{\frac{3}{2}}\left(y_{n}\right)-I_{-\frac{1}{2}}\left(y_{n}\right)=-y_{n}^{-1} I_{\frac{1}{2}}\left(y_{n}\right)
$$

when $a=1 / 2$. We will also repeatedly use the fact that

$$
I_{a}(y) \sim \frac{e^{y}}{\sqrt{2 \pi y}} .
$$

This yields the asymptotic

$$
D_{2}(n) \sim \frac{2}{(24 n-1)^{\frac{1}{4}}} I_{\frac{1}{2}}\left(y_{n}\right),
$$

and since (2.3) and (2.6) show that this difference is smaller than the asymptotic for $M_{2}(n)$ in (2.1), we have also proved that

$$
N_{2}(n) \sim M_{2}(n) .
$$

We next consider the case $k=4$.

Theorem 2.2. As $n \rightarrow \infty$,

$$
\begin{gathered}
M_{4}(n) \sim N_{4}(n) \sim \frac{7 \pi}{120}(24 n-1)^{\frac{5}{4}} I_{-\frac{5}{2}}\left(y_{n}\right) \sim \frac{84}{5} n^{2} p(n), \\
M_{4}(n)-N_{4}(n) \sim \frac{24 \sqrt{6}}{\pi} n^{\frac{3}{2}} p(n) .
\end{gathered}
$$

Corollary 2.3. Garvan's conjecture is true if $k=4$ and $n$ is sufficiently large. 
Proof. Atkin and Garvan's rank-crank PDE [6] formula (5.6) gives the relation

$$
\begin{aligned}
-D_{4}(n) & =\frac{2}{3}(-3 n-1) M_{2}(n)+\frac{5}{3} M_{4}(n)+(-12 n+1) N_{2}(n) \\
& =-D_{2}(n)+\frac{1}{3} M_{2}(n)+12 n D_{2}(n)-14 n M_{2}(n)+\frac{5}{3} M_{4}(n) .
\end{aligned}
$$

We have written the terms in this way because the crank moments can be explicitly written in terms of modular forms, and we (recursively) have bounds for the lower difference function $D_{2}(n)$. Since we aim to show that $D_{4}(n) \sim * n^{3 / 2} p(n)$, Theorem 2.2 allows us to ignore all terms of the form $* D_{2}(n)$ and $* M_{2}(n)$. Among the other terms, the only one that requires additional work is the final summand. From [6], formula (4.7), we know that

$$
\frac{5}{3} \sum_{n} M_{4}(n) q^{n}=\frac{10}{3} P\left(\Phi_{3}+6 \Phi_{1}^{2}\right),
$$

where $P(q):=\prod_{n=1}^{\infty}\left(1-q^{n}\right)^{-1}$ is the partition generating function, and for $j$ odd,

$$
\Phi_{j}(q):=\sum_{n=1}^{\infty} \sigma_{j}(n) q^{n},
$$

with $\sigma_{j}$ being the $j$ th divisor sum. These functions can be written in terms of the classical Eisenstein series

$$
E_{n}(\tau):=1-\frac{2 n}{B_{n}} \Phi_{n-1}(q),
$$

where $n$ is even and $B_{n}$ is the $n$th Bernoulli number.

We next use the Hardy-Littlewood circle method [1] to determine the asymptotic growth coming from (2.8). Let $q:=e^{-2 \pi z}, \operatorname{Re}(z)>0, q_{1}:=e^{-\frac{2 \pi}{z}}$. Then we have the transformation law

$$
\begin{aligned}
& E_{k}(q)=(i z)^{-k} E_{k}\left(q_{1}\right) \quad \text { if } k>2, \\
& E_{2}(q)=(i z)^{-2} E_{2}\left(q_{1}\right)+\frac{6}{\pi z} .
\end{aligned}
$$

In general, if we wish to estimate the coefficients in an expression of the form

$$
\sum_{n} a(n) q^{n}=c P(q) g\left(q_{1}\right) z^{-k}+\ldots,
$$

where $c$ is a constant and $g(q)$ has a holomorphic $q$-series expansion

$$
g(q)=1+\sum_{n>0} a(n) q^{n}
$$

then the asymptotic contribution to $a(n)$ due to the term displayed in (2.10) is

$$
c \cdot 2 \pi(24 n-1)^{\frac{k}{2}-\frac{3}{4}} I_{\frac{3}{2}-k}\left(y_{n}\right) .
$$

This can be seen in the asymptotic formulas of 9 . Again, since our asymptotic aim is $n^{3 / 2} p(n)$, (2.11) implies that we may ignore all terms with weight $k<3$. We first consider

$$
\frac{10}{3} P(q) \Phi_{3}(q)=\frac{10}{3} \frac{P(q)}{240}\left(E_{4}(q)-1\right)=\frac{P(q)}{72}\left(z^{-4} E_{4}\left(q_{1}\right)-1\right) .
$$

By the above considerations, this term contributes

$$
\frac{\pi}{36}(24 n-1)^{\frac{5}{4}} I_{-\frac{5}{2}}\left(y_{n}\right) .
$$


We next consider

$$
20 P(q) \Phi_{1}^{2}(q)=20 \frac{P(q)}{24^{2}}\left(1-E_{2}(q)\right)^{2}=\frac{5}{144} P(q)\left(1+z^{-2} E_{2}\left(q_{1}\right)-\frac{6}{\pi z}\right)^{2} .
$$

The asymptotic contribution from this is

$$
\frac{5}{144}\left(2 \pi(24 n-1)^{\frac{5}{4}} I_{-\frac{5}{2}}\left(y_{n}\right)-24(24 n-1)^{\frac{3}{2}} I_{-\frac{3}{4}}\left(y_{n}\right)\right) .
$$

Thus the first and second terms in the asymptotic expansion of $M_{4}(n)$ are given by the following (the first gives the constants from the statement of the theorem):

$$
\frac{5}{3} M_{4}(n) \sim \frac{5}{3}\left(\frac{7 \pi}{120}(24 n-1)^{\frac{5}{4}} I_{-\frac{5}{2}}\left(y_{n}\right)-\frac{1}{2}(24 n-1)^{\frac{3}{4}} I_{-\frac{3}{2}}\left(y_{n}\right)\right) .
$$

This gives

$$
\begin{aligned}
-D_{4}(n) \sim \frac{24 n}{(24 n-1)^{\frac{1}{4}}} I_{\frac{1}{2}}\left(y_{n}\right)-\frac{56 \pi n^{2}}{(24 n-1)^{\frac{3}{4}}} I_{\frac{3}{2}}\left(y_{n}\right) & +\frac{7 \pi}{72}(24 n-1)^{\frac{5}{4}} I_{-\frac{5}{2}}\left(y_{n}\right) \\
& -\frac{5}{6}(24 n-1)^{\frac{3}{4}} I_{-\frac{3}{2}}\left(y_{n}\right) .
\end{aligned}
$$

The terms involving $I_{\frac{3}{2}}$ and $I_{-\frac{5}{2}}$ are both larger than our claimed asymptotic, and thus we need them to cancel. We rewrite

$$
\frac{7 \pi}{72}(24 n-1)^{\frac{5}{4}} I_{-\frac{5}{2}}\left(y_{n}\right)=\frac{7 \pi(24 n-1)^{2}}{72(24 n-1)^{\frac{3}{4}}} I_{-\frac{5}{2}}\left(y_{n}\right) \sim \frac{56 \pi n^{2}}{(24 n-1)^{\frac{3}{4}}} I_{-\frac{5}{2}}\left(y_{n}\right) .
$$

We now use the relation (2.5) as well as the $a=-3 / 2$ case of (2.4):

$$
I_{-\frac{5}{2}}(y)=I_{-\frac{1}{2}}(y)-\frac{3}{y} I_{-\frac{3}{2}}(y) .
$$

Combining all of this gives

$$
\begin{aligned}
-D_{4}(n) \sim & \frac{24 n}{(24 n-1)^{\frac{1}{4}}} I_{\frac{1}{2}}\left(y_{n}\right)-\frac{5}{6}(24 n-1)^{\frac{3}{4}} I_{-\frac{3}{2}}\left(y_{n}\right) \\
& +\frac{336 n^{2}}{(24 n-1)^{\frac{5}{4}}}\left(I_{\frac{1}{2}}\left(y_{n}\right)-3 I_{-\frac{3}{2}}\left(y_{n}\right)\right) \\
& \sim \frac{-24 \sqrt{6} n^{\frac{3}{2}}}{\pi} p(n) .
\end{aligned}
$$

In particular $M_{4}(n) \sim N_{4}(n)$.

The proof of Theorem 2.2 makes it clear that Conjecture 1.1 reduces to an identity and inequality involving Bernoulli numbers and binomial coefficients that arises by expanding the rank-crank PDE into Eisenstein series and removing all but the highest weight terms.

Proof of Theorem 1.2, The argument for proving the general asymptotics for $M_{k}(n)$ and $N_{k}(n)$ was outlined in the preceding proof of the case $k=4$. Equation (4.8) in [6] states that

$$
\sum_{n} M_{k}(n) q^{n}=c_{k} P(q) \cdot H_{k}(q)
$$

where $H_{k}(q)$ is in the ring generated by $E_{2}, E_{4}, E_{6}$ with rational coefficients, and the highest-weight terms have weight $k$. This is the general case of the identity 
seen in (2.8); the subsequent discussion (including (2.9), (2.10), and (2.11)) implies the correct form of the asymptotic for $M_{k}(n)$.

Finally, the original form of the rank-crank PDE from [6] is not actually written with the term $D_{k}(n)$ as in (2.7), but instead contains the term $N_{k}(n)$ that can easily be expressed as a sum of terms involving $p(n), N_{k^{\prime}}(n)$ and $M_{\ell}(n)$ for $k^{\prime}<k$ and $\ell \leq k$. An inductive argument combined with the asymptotic for $M_{k}(n)$ gives the correct asymptotic form for $N_{k}(n)$.

\section{Concluding Remarks}

Although we have proven the full strength of Conjecture 1.1 for $k=2,4$, using a general computational technique that should allow us to extend the result to any fixed $k$, it would still be appealing to have a combinatorial proof of Garvan's original conjecture. There are at least two such approaches that may be fruitful. First, recall Andrews' definition of $\operatorname{spt}(n)$ as a "weighted" partition function, where each partition of $n$ is counted according to the multiplicity of its smallest part. The fact that $\operatorname{spt}(n)=\frac{1}{2} D_{2}(n)$ suggests that higher $D_{k}(n)$ might also correspond to other weighted partition functions.

A second approach is suggested by striking data that was inspired by observations of Alex Arkhipov during an undergraduate research project supervised by the second author. Define the crank and rank cumulation functions by

$$
\begin{aligned}
\overline{\mathcal{M}}(m, n) & :=\sum_{r \leq m} \mathcal{M}(r, n), \\
\overline{\mathcal{N}}(m, n) & :=\sum_{r \leq m} \mathcal{N}(r, n) .
\end{aligned}
$$

Note that if $m>0$, then by symmetry, $\overline{\mathcal{M}}(m, n)=p(n)-\overline{\mathcal{M}}(-(m+1), n)$ (the same also holds for ranks).

An interesting phenomenon occurs when the cumulation functions are compared. In particular, if $m<0$, then for all $1 \leq n \leq 100$ (tested with MAPLE) we have

$$
\overline{\mathcal{N}}(m, n) \leq \overline{\mathcal{M}}(m, n) \leq \overline{\mathcal{N}}(m+1, n) .
$$

By symmetry, the opposite inequalities hold for $m>0$. This observation may also be stated in terms of ordered lists of partitions. Specifically, for $1 \leq n \leq 100$, there must be some re-ordering $\tau_{n}$ of the partitions $\lambda$ of $n$ such that $|\operatorname{crank}(\lambda)|-$ $\left|\operatorname{rank}\left(\tau_{n}(\lambda)\right)\right|=0$ or 1 , although we do not have an explicit combinatorial description of $\tau_{n}$. If this continues to hold for all $n$, then the Cauchy-Schwarz inequality implies that

$$
D_{2}(n) \leq 2 \sqrt{n} p(n)
$$

which is nearly as good as Theorem 2.1, and similar results would also hold for higher moments. Even if the differences are not all 0 or 1 , as long as they are relatively "small" this approach still would lead to meaningful estimates.

\section{REFERENCES}

1. G.E. Andrews, The theory of partitions, Cambridge University Press, Cambridge, 1998. MR.1634067 (99c:11126)

2. G. E. Andrews, The number of smallest parts in the partitions of $n$, to appear in J. Reine Angew. Math.

3. G. E. Andrews, Partitions, Durfee symbols, and the Atkin-Garvan moments of ranks, Invent. Math. 169 (2007), 37-73. MR2308850 (2008d:05013) 
4. G. E. Andrews and F. Garvan, Dyson's crank of a partition, Bull. Amer. Math. Soc. 18 (1988), 167-171. MR929094 (89b:11079)

5. G. E. Andrews, R. Askey, and R. Roy, Special functions, Encyclopedia of Mathematics and its Applications, 71. Cambridge University Press, Cambridge, 1999. MR.1688958 (2000g:33001)

6. A. O. L. Atkin and F. G. Garvan, Relations between the ranks and the cranks of partitions, Ramanujan Journal 7 (2003), 343-366. MR2035811(2005e:11131)

7. A. O. L. Atkin and H. P. F. Swinnerton-Dyer, Some properties of partitions, Proc. London Math. Soc. 4 (1954), 84-106. MR0060535 (15:685d)

8. K. Bringmann, Aymptotics for rank partition functions, Transactions of the AMS, accepted for publication.

9. K. Bringmann, On the explicit construction of higher deformations of partition statistics, Duke Math. J. 144 (2008), 195-233. MR2437679

10. K. Bringmann, F. Garvan and K. Mahlburg, Partition statistics and quasiweak Maass forms, Int. Math. Res. Not., 2009, no. 1, 63-97.

11. K. Bringmann and K. Ono, Coefficients of harmonic weak Maass forms, preprint.

12. K. Bringmann and K. Ono, Dyson's ranks and Maass forms, to appear in Ann. of Math.

13. K. Bringmann and S. Zwegers, Rank-crank type PDE's and non-holomorphic Jacobi forms, to appear in Math. Res. Lett.

14. F. Dyson, Some guesses in the theory of partitions, Eureka (Cambridge) 8 (1944), 10-15.

15. F. Dyson, Mappings and symmetries of partitions, J. Combin. Theory Ser. A 51 (1989), 169-180. MR1001259 (90f:05009)

16. K. Mahlburg, Partition congruences and the Andrews-Garvan-Dyson crank, Proc. Natl. Acad. Sci. 102 (2005), no. 43, 15373-15376. MR2188922 (2006k:11200)

17. S. Ramanujan, Some properties of $p(n)$; the number of partitions of $n$, Proc. Camb. Phil. Soc. 19 (1919), 207-210.

18. S. Ramanujan, Congruence properties of partitions, Math. Zeitschrift 9 (1921), 147-153. MR 1544457

Mathematical Institute, University of Cologne, Weyertal 86-90, 50931 Cologne, GERMANY

E-mail address: kbringma@math.uni-koeln.de

Department of Mathematics, Massachusetts Institute of Technology, Cambridge, MASSACHUSETTS 02139-4307

E-mail address: mahlburg@math.mit.edu 